\title{
Anesthetic consideration for patients with severe tracheal obstruction caused by thyroid cancer -A report of 2 cases-
}

\author{
Joong Woon Cho, Mi Ae Jeong, Jin Hwa Choi, Joo Won Cho, Hee Jong Lee, Dong Won Kim, \\ Kyo Sang Kim, and Jung Kook Seo \\ Department Anesthesiology and Pain Medicine, Hanyang Medical Center, Hanyang University College of Medicine, Seoul, Korea
}

To achieve safe airway management, it is essential first to predict whether there will be difficulties in intubating or ventilating the patient's airway. An enlarged thyroid mass can produce a tracheal obstruction by compression or intraluminal invasion or both. We report two patients with thyroid cancer that obstructed the trachea by compression or invasion. There was no difficulty in endotracheal intubation of the patients with marked thyroid enlargement or in securing passage of the endotracheal tube through the compressed or narrowed portion of the trachea. (Korean J Anesthesiol 2010; 58: 396-400)

Key Words: Difficult intubation, Thyroid cancer, Tracheal obstruction.

There are several causes of a tracheal obstruction, of which metastasis or compression by adjacent tumors is most common [1]. An upper respiratory tract obstruction caused by the compression of tumors adjacent to the airway outside the thorax, particularly thyroid cancer, shows considerable mobility and make possible a larger tracheal tube for intubation than the measured inside diameter (ID) [2-8]. Although there are reports showing that a tracheal obstruction caused by thyroid cancer does not create too much difficulty in endotracheal intubation and allows the intubation of a larger sized endotracheal tube than the measured ID [2,6-8], no such clinical findings have been reported in Korea. All Korean case studies employed special methods, such as percutaneous extracorporeal circulation, cardiopulmonary bypass, epidural anesthesia, etc
$[5,9,10]$. This paper reports two cases of successful anesthesia using an endotracheal tube with a larger ID than that measured by computerized tomography (CT) in patients with a tracheal obstruction caused by thyroid cancer, and discusses the appropriate treatments related to the causes or locations of the tracheal obstruction.

\section{Case Report}

\section{Case 1}

A 72 year-old, $150 \mathrm{~cm}, 57 \mathrm{~kg}$, female patient presented with a neck mass that developed a few years earlier. She had no specific medical records except for taking medication for

Received: June 2, 2009. Revised: 1st, June 16, 2009; 2nd, June 22, 2009. Accepted: July 8, 2009.

Corresponding author: Mi Ae Jeong, M.D., Department Anesthesiology and Pain Medicine, Hanyang Medical Center, Hanyang University College of Medicine, 17, Haengdang-dong, Seongdong-gu, Seoul 133-792, Korea. Tel: 82-2-2290-8680, Fax: 82-2-2299-8692, E-mail: macheong@hanyang.ac.kr (ㄷ) This is an open-access article distributed under the terms of the Creative Commons Attribution Non-Commercial License (http:// creativecommons.org/licenses/by-nc/3.0/), which permits unrestricted non-commercial use, distribution, and reproduction in any medium, provided the original work is properly cited. 
hypertension for 2 years. A physical examination revealed a palpable, broad, and firm tumor around both thyroid glands, but she did not complain of any symptoms. Neck CT detected a thyroid neoplasm on both sides (about $6 \times 3 \mathrm{~cm}$ on the right thyroid gland; about $7 \times 7 \mathrm{~cm}$ on the left thyroid), which revealed the presence of tracheal stenosis. The region of tracheal constriction narrowed from $3.6 \mathrm{~mm}$ below the glottis to the sternal notch, in which the narrowest lumen of the trachea was approximately $3.9 \times 21 \mathrm{~mm}$ in diameter and appeared to be compressed by the tumors of both sides (Fig. 1). Intratracheal metastasis was not suspected. A thyroid papillary carcinoma was diagnosed by an aspiration biopsy, and on thoracic CT, a concern of metastasis into the lung urged immediate treatment. Arterial blood gas analysis (ABGA) showed a pH of 7.424, $\mathrm{PaCO}_{2}$ of $43.1 \mathrm{mmHg}, \mathrm{PaO}_{2}$ of $74.8 \mathrm{mmHg}$, and Base Excess of 2.9. A pulmonary function test was not performed due to the patient's persistent refusal. After admission, diabetes was diagnosed and insulin treatment was started. The patient was scheduled for a thyroid resection and radical neck dissection. The distance between the glottis and constriction site was so short that an endotracheal tube could not to be placed above the constriction region. A tracheostomy was not possible because the tumor covered the site. However, there were no episodes of respiratory disturbance despite the severe tracheal obstruction, nor was there any increase in the $\mathrm{PaCO}_{2}$. The partial pressure of oxygen $\left(\mathrm{PaO}_{2}\right)$ also was in the normal range for her age. Upon auscultation, normal breathing sounds were heard, which made us suspect that the tracheal obstruction was mobile. After explaining the potential difficulty of endotracheal intubation due to the tracheal stenosis one day before surgery, the patient provided informed consent for the procedure. A flexible fiberoptic bronchoscopy and laryngeal mask airway (LMA) were prepared, and uncuffed endotracheal tubes, 4.0, 4.5,

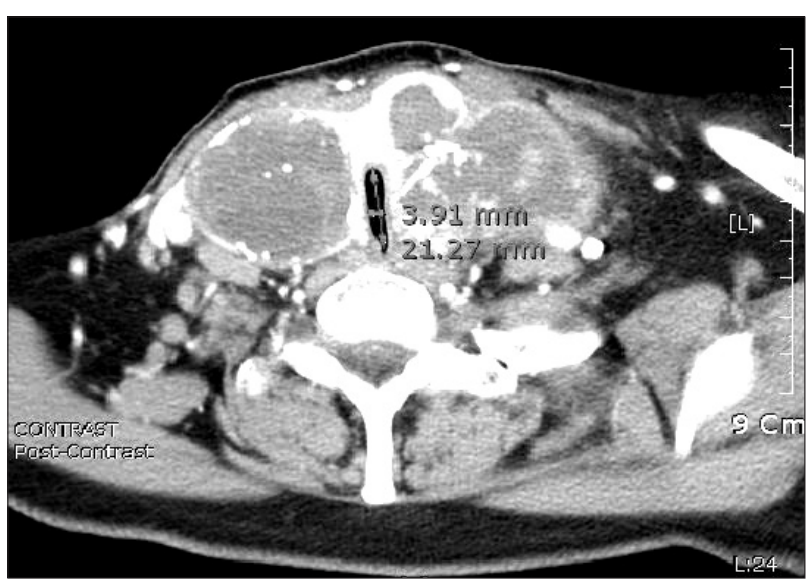

Fig. 1. Computed tomography scan showing a marked thyroid mass compressing the trachea. The narrowest lumen of the trachea was 3.9 $\times 21.2 \mathrm{~mm}$ in diameter.
5.0 in size, and cuffed endotracheal tubes, 4.0 to 7.0 in size, were ready for use. For pre-anesthesia medication, $0.5 \mathrm{mg}$ of atropine was administrated 30 minutes before the onset of anesthesia. An electrocardiogram (ECG), non-invasive blood pressure (NIBP) monitor, and pulse oximetry were placed after arriving in the operating room. Her vital signs indicated a BP, heart rate and oxygen saturation of 145/85 $\mathrm{mmHg}, 92 / \mathrm{min}$ and $99 \%$, respectively, and she did not complain of any discomfort in the supine position. Before the onset of anesthesia, denitrogenation was induced with $100 \%$ oxygenation at $6.0 \mathrm{~L} / \mathrm{min}$ for 5 minutes. Because she had no dyspnea during sleep, we tried to attempt endotracheal intubation using bronchoscope during sleep. Midazolam $3 \mathrm{mg}$ was administered and the patient fell asleep. However, when a bronchoscope intubation was attempted, her spontaneous respiration stopped and support ventilation was provided. When assisted ventilation with $100 \%$ oxygen was confirmed to function well, thiopental $100 \mathrm{mg}$ was administered and the ventilation function was reconfirmed. Therefore, the possibility of using a laryngoscope rather than a bronchoscope was considered. Succinylcholine $50 \mathrm{mg}$ was then administrated and endotracheal intubation was attempted. After inserting an uncuffed endotracheal tube of 4.0 without resistance, the tube was withdrawn while keeping the bronchoscope in place. When a cuffed endotracheal tube (size 5.0) was inserted without difficulty, the tube was replaced smoothly with a cuffed endotracheal tube (size 6.5). Rocuronium $30 \mathrm{mg}$ was then injected into the vein and anesthesia was maintained with both oxygen and nitrous oxide of $1.5 \mathrm{~L} / \mathrm{min}$, and sevoflurane $1.0-$ 2.5 vol\%, while remifentanil was administrated continuously as a supplement. The tidal volume (TV), respiratory rate (RR) and peak inspiratory pressure (PIP) was $500 \mathrm{ml}, 10 / \mathrm{min}$, and $18 \mathrm{cmH}_{2} \mathrm{O}$, respectively. The operation was started after placing a catheter into the left dorsalis pedis artery for continuously monitoring of the arterial blood pressure and ABGA. Eight hours and 40 minutes after its onset, the operation was completed uneventfully. Extubation was performed after confirming that the patient's spontaneous breathing was restored and she had reached consciousness. She was transferred to the Ward after sufficient monitoring in the post-anesthesia recovery unit.

\section{Case 2}

A 66 year-old, $175 \mathrm{~cm}, 73 \mathrm{~kg}$, male patient was referred to our institution from a clinic due to an episode of dyspnea over the last 3-4 months. He was diagnosed with bronchial asthma and received medical treatment, but there were no favorable changes and he was admitted to our hospital. He had no other abnormalities in his medical records other than taking medication due to a 20 -year history of hypertension. He often experienced respiratory disturbances in the left 
lateral decubitus or supine position, whereas there were no complaints of dyspnea in the right lateral decubitus or semisupine position. A physical examination revealed a firm tumor, approximately $3 \mathrm{~cm}$ in size, below the right thyroid, but a simple chest X-ray did not show any abnormalities. The ABGA results were $\mathrm{pH} 7.413, \mathrm{PaCO}_{2} 44.0 \mathrm{mmHg}, \mathrm{PaO}_{2} 106.4 \mathrm{mmHg}$, and Base Excess 2.4. The pulmonary function test showed mild lung impairment with a mild obstructive pattern (FVC $3.8 \mathrm{~L} / \mathrm{min}$ [89.4\% of predicted value], FEV1 $2.53 \mathrm{~L} / \mathrm{min}$ [93\% of predicted value]). Cervical magnetic resonance imaging (MRI) detected an ill-defined tumor in the lower part of the right thyroid and showed that the tumor had invaded the trachea and formed a polypoid tumor in the postro-internal direction. The tumor was distributed from approximately $2-3 \mathrm{~cm}$ below the glottis to the sternal notch with the narrowest region being $2.79 \mathrm{~mm}$ in diameter at approximately $4 \mathrm{~cm}$ below the glottis (Fig. 2), indicating a metastasis of the thyroid cancer into the trachea. A partial resection of the thyroid and trachea was determined after diagnosing thyroid papillary carcinoma by an aspiration biopsy.

The distance from the glottis was so short that a tracheal tube was not placed above the region of the tracheal obstruction. A tracheostomy was not possible because the tumor had already invaded the sternal notch. Extracorporeal circulation was planned under the suspicion that a metastasis of the tumor into the trachea would cause a high airway pressure, and extracorporeal circulation would be safer than laryngeal mask airway due to the symptoms of dyspnea. After consultation regarding our plan, his attending surgeon strongly recommended endotracheal intubation on the grounds that the base of the polypoid legion was so broad that the tissue would be far from being damaged or cut off and that there would be no bleeding or necrosis. Therefore, it was decided to attempt endotracheal intubation through a bronchoscope under the

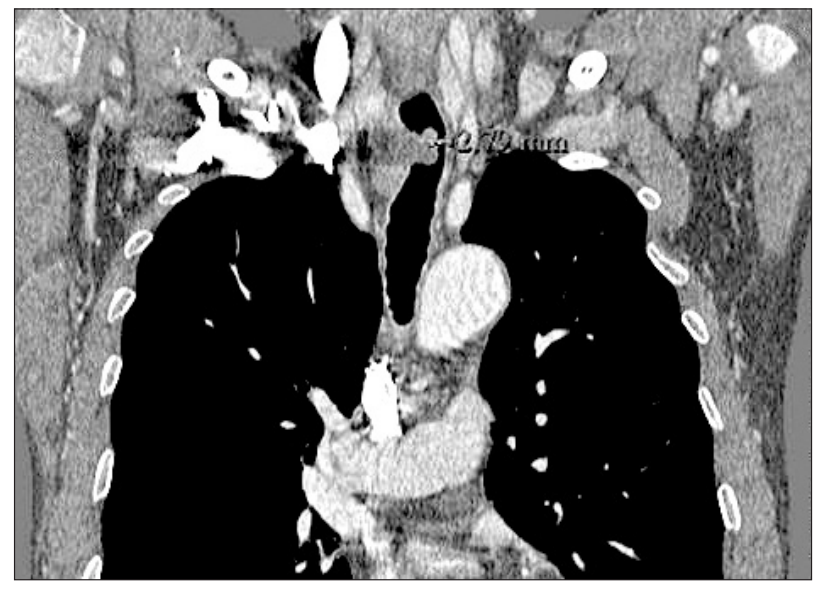

Fig. 2. MRI scan shows a marked tracheal obstruction caused by thyroid cancer invasion. The narrowest portion of the trachea was $2.79 \mathrm{~mm}$ in diameter. sleep state. In case of failure of endotracheal intubation, a LMA was provided and endotracheal tubes (size 4.0 and 4.5 without a cuff and sizes 4.0 to 7.5 with a cuff) were set. One day before the operation, the patient was given an explanation of the potential difficulty in endotracheal intubation due to the presence of the tumor inside the trachea, the process of intubation under conscious sedation or in the awakened state, and the possibility of extracorporeal circulation. The patient provided informed consent for the procedure. There was no pre-operative medication and the upper body was elevated to approximately $30^{\circ}$ after arriving in the operating room. The BP measured immediately after entering the OR was 180/112 $\mathrm{mmHg}$, which necessitated the administration of hydralazine $10 \mathrm{mg}$. A catheter was placed into the right dorsalis pedis artery to monitor the arterial BP and perform ABGA. Propofol and remifentanil were administered continuously for sedation to maintain normal breathing, and oxygenation was provided by a mask. When respiration became shallow, assisted ventilation was performed and the ventilation rate was increased until the patient's respiration had returned to normal, and reached a proper sleep state and did not respond to hearing stimulus. A size 5.0 cuffed endotracheal tube was threaded over a $4.0 \mathrm{~mm}$ flexible bronchoscope and the bronchoscope was inserted carefully into the region of the tumor. Although there was a cough attack when the bronchoscope was passed over the glottis, the bronchoscope was advanced uneventfully up to the tumor location in a single attempt. After confirming the tumor, the bronchoscope was advanced further to confirm the carina. To prevent damage to the tumor, the tracheal tube threaded over the bronchoscope was advanced with a $180^{\circ}$ turn in order for its bevel to be directed toward the right. Thiopental 150 mg and vecuronium $8 \mathrm{mg}$ were administered after tracheal intubation. The length of the 5.0 tracheal tube was $22 \mathrm{~cm}$, and it was pushed up as much as possible to allow the adapter part of the tube to be attached to the patient's incisor. The tip of the tube was confirmed to be placed above the carina. Before removing the bronchoscope, we checked for any damaged tissue, and after removing it, suction was performed twice to remove the unfound tissue debris. Mannual ventilation was provided with $100 \%$ oxygen at $4 \mathrm{~L} / \mathrm{min}$ and sevoflurane, using the anesthesia machine. A cuffed 6.0 endotracheal tube was inserted through a tube exchanger because the PIP increased sharply to $35 \mathrm{cmH}_{2} \mathrm{O}$ and the length of the tube was too short to be advanced further on resection of a part of the trachea. A bronchoscope was inserted to again confirm the removal of fragments due to tumor damage, followed by further suction. No fragments of damaged tissue were found, and the administration of protofol was ceased. Anesthesia was maintained with both $500 \%$ oxygen and nitrous oxide $1.5 \mathrm{~L} /$ min, respectively and sevoflurane supplemented with the 
continuous administration of remifentanil. The operation proceeded and was complete 7 hours after its onset. The patient was transported to the intensive care unit with an endotracheal tube. Later, he was discharged home without complications.

\section{Discussion}

A tracheal obstruction has many symptoms according to the location, degree and cause, and is generally treated according to the symptoms. The airway obstruction is caused by primary tumors in the trachea, or secondarily by compression on the trachea or metastasis into the trachea by a neighboring tumor or by granuloma growth from a previous history of tracheal intubation, injury, or inflammation [11-13]. From the location, tracheal obstructions are divided into two groups: obstructions inside the thorax and obstructions outside the thorax. In the case of obstructions inside the thorax, it is essential to determine if the obstruction occurs in the trachea or in the bronchus. Regardless of the cause, a bronchial obstruction is less life-threatening than a tracheal obstruction. A tracheal obstruction in the posterior mediastinum caused by the compression of a tumor inside the thorax does not carry a large risk. On the other hand, an obstruction in the anterior mediastinum may impede the maintenance of proper ventilation by increasing the compression on the trachea by the tumor when the patient's spontaneous respiration disappears or muscle relaxation is achieved [14]. Tumors in the anterior mediastinum require a prompt warning of severe post-general anesthesia complications if measurements of the peak expiratory flow rate and tracheal area are $<50 \%$ of the expected one [15]. In the case of a tracheal obstruction outside the thorax, it is essential to confirm whether it is caused by neighboring tumors, intratracheal tumors, or scars resulting from trauma or inflammation. A tracheal obstruction caused by trauma or inflammation, regardless of its position, allows only endotracheal tubes with a smaller size than the measured diameter. Therefore, it is essential to make an accurate estimate of the extent of tracheal obstruction. In cases of difficult endotracheal intubation due to a severe obstruction, the anesthetic method should be decided according to whether the obstruction site is above or below a possible site of the tracheostomy. A tracheal obstruction can also be caused by tissue fragments from a primary intratracheal tumor during or after tracheal intubation. Although such obstructions are rare, it is important to understand the features and characteristics of tumors even in smaller ones. Bouaggad et al. [6] reported that a huge thyroid tumor did not raise the issue of difficult endotracheal intubation (DEI), and some cases with DEI did not show any difficulty when the endotracheal tube was passed over the compressed region [6-8]. By contrast, the degree of thyroid tumor progression is a more dangerous factor for DEI because fibroblastic proliferation caused by the progression of thyroid cancer decreases the mobility of the larynx [6].

Regarding the anesthetic methods for patients with tracheal obstruction, there are several options worth considering. These include placement of a tracheal tube above the tracheal obstruction site, placement of a tube with a smaller diameter being passed over the obstruction site, tracheostomy, use of a LMA, high frequency jet ventilation, high frequency positive pressure ventilation, extracorporeal circulation, etc. All these methods can secure the airway, but each method is vulnerable to certain complications. Therefore, careful consideration should be given when choosing the most suitable method. In any patient in whom a tracheal obstruction is suspected, anesthesia should be induced with the preparation of all necessary tools and equipment ready in case of emergency.

In the present cases, thyroid cancer obstructed the trachea by compression or invasion. In case 1, the tumor grew in both thyroid glands and compressed the trachea on both sides; the narrowest diameter of the trachea was approximately $3.9 \times$ $21.2 \mathrm{~mm}$. However, the patient did not complain of dyspnea in any position. ABGA revealed normal findings, whereas the trachea with an anterioposterior diameter of approximately $21.2 \mathrm{~mm}$ was compressed by the tumor on both sides but the thyroid tumor was believed not to be fixed to the surrounding tissues and was mobile. Her Surgeon stated that a tracheotomy under local anesthesia would be difficult because the tumor was large enough to cover the tracheotomy site. Taking the use of LMA or extracorporeal circulation into account, the relevant clinical reports were reviewed, which showed that endotracheal tubes with diameter a larger than the tracheal diameter could inserted in cases of a tracheal obstruction caused by the compression of thyroid cancer [2-8]. Therefore, a flexible fiberoptic bronchoscope, LMA, and a variety of endotracheal tubes of different sizes were made ready in case of emergency, and the induction of general anesthesia was scheduled after making preparations for an otolaryngologist to perform an emergency tracheostomy. Although tracheal intubation under consciousness is safer, the patient did not have any symptoms of dyspnea during sleep, so a sleep state was induced with midazolam, and we tried to attempt tracheal intubation during sleep under a bronchoscope. Manual ventilation was needed due to the occurrence of apnea during the induction of sleep, and tracheal intubation was performed under the direct laryngoscope. The tracheal obstruction was so severe that the endotracheal tubes were changed gradually to a larger size from $4.0,5.0$, to 6.5 , while checking the airway resistance to reduce the potential damage caused by intubation. In retrospect, it may have been worth attempting to insert a 6.5 size tube next to a 4.0 size one to save time and trouble. As expected, an endotracheal 
tube with a diameter larger than the measured inside diameter (ID) was easily inserted. In this case, a partial tracheostomy was not scheduled because there were no signs of endotracheal metastasis, so LMA was not employed because it was believed that it would not be able to maintain anesthesia for more than 6 hours until the thyroid tumor had been removed completely. However, a plan for LMA and tracheostomy was made as a contingency plan for difficult endotracheal intubation (DEI). Extracorporeal circulation also was not attempted due to the risk of blood loss because this method was rather invasive and required heparin despite its advantage of enabling anesthesia without touching the trachea when it is difficult to secure the airway.

In case 2 , the thyroid cancer invaded the trachea. The most severe obstruction was located approximately $3 \mathrm{~cm}$ below the glottis. Its diameter was approximately $2.8 \mathrm{~mm}$, and the tumor formed a polypoid mass. To avoid damage to the tumor, a 4.0 $\mathrm{mm}$ flexible fiberoptic bronchoscope was inserted through a cuffed 5.0 size endotracheal tube to reduce the space between the bronchoscope and tube while a 6.5 size endotracheal tube was inserted for replacement with its bevel being directed toward the tumor lesion. There was no tumor tissue debris found on the gross examination (Fig. 3), but the anesthetic method in case 2 did not appear to be the proper choice in retrospect. This is because naked eye observation does not ensure that there is no tumor tissue debris remaining and the presence of such fragments may be another issue of bronchial obstruction. The reason why the method in this case did not present any problems is that the endotracheal tube with a diameter larger than the measured ID could be inserted because the trachea compressed by the thyroid tumor was not

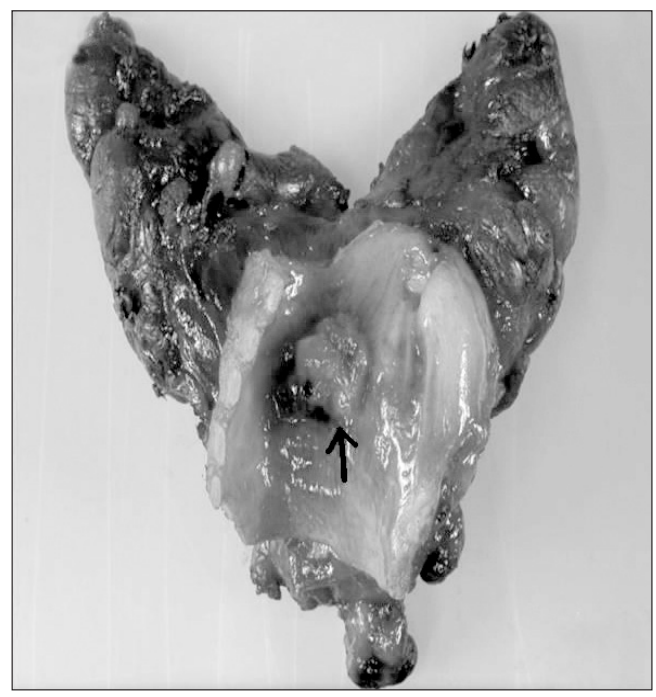

Fig. 3. This figure shows the resected partial trachea and thyroid gland. The arrow indicates an intratracheal polypoid mass. constricted by foreign surrounding tissue.

In conclusion, endotracheal tubes with a diameter larger than the measured ID were used despite the severe tracheal obstruction due to the absence of dyspnea or very mild symptoms in patients showing normal ABGA and pulmonary function test results. In addition, the normal results of these two tests demonstrated that the trachea compressed by the foreign tumor outside the thorax was mobile. Several clinical and research papers support our reasoning [2-8]. As suggested in our cases, anesthesia should be initiated only after making all preparations for securing the trachea, even when easy tracheal intubation is anticipated. As in case 2, it is safer to perform tracheal intubation in the awakened state.

\section{References}

1. Wood DE. Management of malignant tracheobronchial obstruction. Surg Clin North Am 2002; 82: 621-42.

2. Weinbroum AA, Kidron A, Mohr R, Kudlik N, Gil Z, Fliss DM. Multidisciplinary management of life-threatening tracheal obstruction. Resuscitation 2005; 64: 115-7.

3. Toyota K, Uchida H, Ozasa H, Motooka A, Sakura S, Saito Y. Preoperative airway evaluation using multi-slice three-dimensional computed tomography for a patient with severe tracheal stenosis. Br J Anaesth 2004; 93: 865-7.

4. Mizuno J, Nakano M, Kasuya M, Nishiyama T, Hanaoka K. A case of giant thyroid tumor with tracheal stenosis. Masui 2004; 53: 682-6.

5. Yoo TJ, Lim YH, Lee SS, Yoo BH, Woo SH, Yon JH. Percutaneous cardiopulmonary support for tracheal stenosis caused by thyroid mass. Korean J Anesthesiol 2008; 54: 703-7.

6. Bouaggad A, Nejmi SE, Bouderka MA, Abbassi O. Prediction of difficult tracheal intubation in thyroid surgery. Anesth Analg 2004; 99: 603-6.

7. McHenry CR, Piotrowski JJ. Thyroidectomy in patients with marked thyroid enlargement: airway management, morbidity, and outcome. Am Surg 1994; 60: 586-91.

8. Shaha AR, Burnett C, Alfonso A, Jaffe BM. Goiters and airway problems. Am J Surg 1989; 158: 378-80.

9. Kwon JY, Lee HJ, Kim HK, Baik SW. Femorofemoral cardiopulmonary bypass in the management of severe airway obstruction by thyroid cancer. Korean J Anesthesiol 2004; 46: 236-40.

10. Kook EY, Ahn YM, Park CH, Lee C, Lee CS, Kim WT. Cervical epidural anesthesia for thyroidectomy in a patient with tracheal deviation. Korean J Anesthesiol 1999; 37: 159-63.

11. Finlayson GN, Brodsky JB. Anesthetic considerations for airway stenting in adult patients. Anesthesiol Clin 2008 26: 281-91.

12. Pinsonneault C, Fortier J, Donati F. Tracheal resection and reconstruction. Can J Anaesth 1999; 46: 439-55.

13. Sandberg W. Anesthesia and airway management for tracheal resection and reconstruction. Int Anesthesiol Clin 2000; 38: 55-75.

14. Morikawa N, Honna T, Kuroda T, Tanaka K, Kitano Y, Nakano M, et al. Life-threatening airway obstruction caused by mediastinal germinoma in a 9-year-old girl. J Pediatr Surg 2005; 40: 588-90.

15. Ricketts RR. Clinical management of anterior mediastinal tumors in children. Semin Pediatr Surg 2001; 10: 161-8. 